\title{
Ambiente educativo en la escuela de medicina de una Universidad pública: diagnóstico basal
}

\section{Educational environment in the medical school of a public University: Baseline diagnosis}

\author{
Laura-V Torres ${ }^{1}$; Argénida Blanco-Gómez ${ }^{1}$ \\ Forma de citar: Torres LV, Blanco-Gómez A. Ambiente educativo en la escuela de medicina de una \\ Universidad pública: diagnóstico basal. Rev Univ Ind Santander Salud UIS. 2018; 50(4): $342-349$. \\ doi: http://dx.doi.org/10.18273/revsal.v50n4-2018007 (c) (1)
}

\section{Resumen}

Introducción: La evaluación del ambiente educativo en medicina proporciona pautas para realizar cambios curriculares enfocados a lograr un mejor desempeño del estudiante y futuro profesional. En el presente estudio participaron de forma voluntaria 490 estudiantes de medicina de la Universidad Industrial de Santander. Objetivo: Medir el ambiente educativo, mediante el cuestionario DREEM (50 preguntas que evalúan cinco dimensiones: percepción del aprendizaje, percepción hacia los docentes, autopercepción académica, percepción del ambiente y autopercepción social), siendo el instrumento más utilizado para medir la calidad de la educación médica en el mundo. Resultados: El principal hallazgo encontrado fue la disminución drástica en la percepción positiva hacia los docentes a medida que se avanzó en la carrera, resaltando que al inicio los estudiantes prefieren no emitir juicios. Con respecto a las dimensiones de percepción de aprendizaje y autopercepción académica se encontraron los valores más bajos en tercer y sexto año de carrera. En cuanto a las actividades extracurriculares, el asistir a culto religioso y tocar un instrumento musical, fueron las que menos variación presentaron, comparando antes y después de ingresar a la escuela de Medicina de la Universidad Industrial de Santander. Conclusión: Este estudio pretende ser herramienta útil para replantear la estructura y detectar probables debilidades del programa de medicina de pregrado en la UIS, con el objetivo de generar recomendaciones a directivos, que permitan proyectar al estudiante de medicina como un ser integral y propender por el desarrollo armónico de sus dimensiones, en la que el aspecto académico sea considerado con igual importancia como el tiempo dedicado al descanso y a la practica de actividades extracurriculares, que le garanticen aprender sin afectar su calidad de vida.

Palabras clave: Educación médica; Ambiente educativo; DREEM.

\begin{abstract}
Introduction: The evaluation of the educational environment in medicine provides guidelines for restructuring curricular changes aimed to achieve at achieving a better performance of the student and future professional. In the present study were involved 490 medical students from the Universidad Industrial de Santander. Objetive: To measure the educational environment with the DREEM questionnaire (50 questions about five aspects: perception of learning, perception of teachers, academic self-perception, perception of the environment and social self-

1. Universidad Industrial de Santander. Bucaramanga, Colombia.

Correspondencia: Argénida Blanco Gómez. Dirección: Carrera 32 29-31. Facultad de Salud UIS Bucaramanga. Teléfono: +5776344000. Correo electrónico: argenida@uis.edu.co.
\end{abstract}


perception). We decided to use the DREEM because it's being the most common survey to gauge the quality of medical education. We found an important diminution in the positive perception of teachers, throughout with the pass of the years of $\mathrm{n}$ the medical school. About academic self-perception and learning perception the lowest values were found at the third and sixth year of medical school. Finally the most common activities that medical students kept doing during the years of medical career were the religious and musical activities.

Keywords: Medical education; Educational environment; DREEM.

\section{Introducción}

El proceso de enseñanza-aprendizaje de la medicina enfrenta grandes retos debido al contexto cambiante, tanto global como local. Hay una tendencia mundial de superar el modelo biomédico clásico hacia uno más centrado en la persona y su entorno, percibiéndolo como un ser integral, imponiéndose como un medio de adaptarse a las nuevas realidades sociales ${ }^{1,2}$. A esto se suma que el sistema de salud colombiano enfatiza cada vez mas en la promoción de la salud, prevención de la enfermedad y atención primaria, por lo cual se requiere de manera urgente que los nuevos médicos tengan competencias adecuadas para este entorno laboral $^{3}$. Esto se ha manifestado en un listado de 104 recomendaciones hechas por una comisión ad hoc de expertos en educación médica ${ }^{4}$. que posteriormente fue acogida por diversas escuelas adscritas a la Asociación Colombiana de Facultades de Medicina en el llamado Consenso de Montería ${ }^{5}$.

En los últimos años, la Escuela de Medicina de la Universidad Industrial de Santander (UIS) en consonancia con este contexto ha estado discutiendo la necesidad de una reforma curricular y de cambios en los enfoques pedagógicos utilizados. Para ello la Oficina de Educación Médica apoyada por el Comité Curricular y la Dirección de la Escuela, decidió monitorear el ambiente educativo de los estudiantes de medicina de la UIS, como una forma de tener una herramienta objetiva para apoyar la toma de decisiones relacionadas en el programa de medicina.

El ambiente educativo puede ser entendido como la sumatoria de impresiones, creencias y expectativas que los miembros de una comunidad educativa tienen de su propia escuela, las conductas asociadas, los símbolos y estamentos que representan dichas conductas ${ }^{6}$. Estos elementos son importantes para cada individuo que forma parte del programa de capacitación ya sea de pregrado, postgrado o educación continua, puesto que de ellos depende la calidad del aprendizaje, respeto por la enseñanza y confianza en los profesores. Si ésto ocurre, la motivación de los alumnos no será un problema y el producto final implicará mejores profesionales.
Entre las variables que tienen impacto en la percepción del ambiente, se encuentran las habilidades de los profesores, aulas de clase, duración de clases, tipo de materiales de aprendizaje, métodos de enseñanza, estudio, evaluación, biblioteca, instalaciones de ocio, acceso a computadores, entre otros ${ }^{6}$. Resaltando como componentes clave para un adecuado clima educativo: Interacción con profesores, número de materias, método de enseñanza ${ }^{7}$.

El estudio del ambiente educativo guarda una relación directa de forma positiva, con el rendimiento académico y personal del alumno, administrativos y docente, brindando herramientas a las instituciones para introducir cambios dirigidos a la excelencia. En el momento existen alrededor de 30 escalas validadas para medir el ambiente educativo, siendo la mas utilizada la escala DREEM (Dundee Ready Education Enviroment Measure) en pregrado de medicina, por el enfoque cualitativo que implica la recopilación de datos principalmente a través de exploración de la experiencia de aprendizaje ${ }^{6}$. Siendo el DREEM el de nuestro interés por su gran confiabilidad estadística con el propósito de generar un perfil institucional, para compararnos con otras escuelas de medicina y conocer la percepción del entorno educativo. El objetivo de este estudio es hacer un diagnóstico inicial del ambiente educativo de la Escuela de Medicina de la Universidad Industrial de Santander, permitiendo detectar situaciones a mejorar, que requieran intervención y que sirva de base para futuras mediciones que hagan posible identificar cambios necesarios al currículo y prácticas pedagógicas, para mejorar el aprendizaje.

\section{Materiales y métodos}

Se aplicó el DREEM a 490 estudiantes, de los 595 que aparecen matriculados en el programa de Medicina de la Universidad Industrial de Santander; con datos administrativos propios de la Dirección de Educación Médica, que contaron con anonimato y confidencialidad. Inicialmente se planteó incluir a todos los estudiantes de pregrado matriculados en el primer semestre de 2018, aunque en la práctica sólo participaron quienes estuvieron en las clases teóricas en las que se hizo la 
recolección de datos y decidieron libremente participar. Para tal fin una de las investigadoras acordó con los profesores un momento al final de la clase en el que se aplicó un instrumento con datos de sexo, edad $\mathrm{y}$ año de estudio, unas preguntas sobre actividades extracurriculares realizadas antes de ingresar a estudiar medicina y los cambios de frecuencia en su realización durante los estudios; éstas fueron leer libros, asistir a misa o culto religioso, jugar en el computador, salir a fiestas o conciertos, asistir a cine, practicar deporte, salir de paseo, salir a hacer compras, tocar instrumento musical y ver televisión.

Ambiente educativo. Se usó una adaptación del Dundee Ready Education Environment Measure (DREEM), basada en las versiones en español adaptadas por Aguilar-Barojas, et al. $^{7}$ y Riquelme, et al. ${ }^{8}$ Estas versiones fueron validadas mediante procesos descritos en detalle en otras publicaciones con estudiantes de pregrado de la Universidad Juárez Autónoma de Tabasco en México $^{7}$ y la Universidad Pontificia Universidad Católica de Chile $^{8}$. La versión final usada en este estudio mantuvo las cinco subescalas o dimensiones del ambiente educativo, con sus respectivos ítems: i) percepción del aprendizaje (ítems: 1, 7, 13, 16, $20,22,24,25,38,44,47$ y 48), ii) percepción de los docentes (ítems: 2, 6, 8, 9, 18, 29, 32, 37, 39, 40 y 50), iii) autopercepción académica (ítems: 5, 10, 21, 26, 27, 31, 41 y 45), iv) percepción del ambiente (ítems: $11,12,17,23,30,33,34,35,36,42,43$ y 49), y v) autopercepción social (ítems: 3, 4, 14, 15, 19, 28 y 46). Todos los ítems tuvieron las siguientes cinco opciones de respuesta: total desacuerdo (1), en desacuerdo (2), indeciso (3), en acuerdo (4) y total acuerdo (5).

El puntaje final de cada dimensión correspondió a la sumatoria de los ítems, teniendo en cuenta que los ítems 4, 8, 9, 17, 25, 35, 39, 48 y 50 estaban invertidos. De esta manera los valores mínimos y máximos de cada dimensión fueron: percepción del aprendizaje de 12 a 60, percepción de los docentes de 11 a 55, autopercepción académica de 8 a 40, percepción del ambiente de 12 a 60 , y autopercepción social de 7 a 35, siendo mejor el ambiente educativo cuando mayores valores se obtienen.

\section{Métodos estadísticos}

La descripción de las variables y de las subescalas del DREEM se hizo mediante porcentajes y medidas de tendencia central y dispersión. La comparación entre los diferentes años de estudio y los puntajes obtenidos en las dimensiones del DREEM se hizo mediante las pruebas de Kruskal-Wallis o t de Student, después de verificar si se tenía o no distribución normal con la prueba de Shapiro-Wilk. La consistencia interna de cada dimensión fue valorada con alfa de Cronbach. Para el análisis se usó el programa estadístico Stata 14.

Listados libres. Para identificar las asignaturas consideradas como más difíciles y las que más eran reprobadas, se pidió que los estudiantes las listaran. Para su análisis se usó la técnica de listados libres, con el que se obtiene un valor de Salience de Smith entre cero y uno, que en su construcción considera la frecuencia con que aparece cada respuesta y la posición relativa en la que es listada cada respuesta9 ${ }^{9}$. A mayor valor es más relevante la respuesta dentro del conjunto de asignaturas de pregrado de medicina. Este análisis se hizo con el software Anthropac $4.983^{10}$.

\section{Resultados}

En la encuesta participaron 490 estudiantes; en la Tabla 1 se encuentra la proporción de participantes por año de estudio. En comparación con el número de matriculados, la participación puede considerarse aceptable (490), correspondiendo al $82.35 \%$ del total de estudiantes matriculados (595). Como se puede apreciar en la Tabla 1, la mayor participación estuvo en cuarto año y la menor en tercer año, lo cual puede responder a la disponibilidad de estar en el momento de recolección de datos. Tanto el sexo como la edad de los participantes sugieren que hubo una buena representación de los estudiantes. La percepción inicial es que estudiar medicina en la UIS es más difícil que otras carreras, y más difícil que en otras universidades.

En la Tabla 2 se encuentran los puntajes obtenidos en las dimensiones del ambiente educativo. Un primer hallazgo es que los estudiantes de los dos primeros años no quisieron brindar información sobre los docentes. Todos los puntajes estuvieron por encima del valor intermedio, sin sobrepasar el $75 \%$ del valor máximo posible, lo que puede interpretarse como una percepción aceptable de los ambientes educativos. Sin embargo, resultó muy llamativo que entre los 6 años de estudio se observaran diferencias significativas en todas las dimensiones; la tendencia en las dimensiones de percepción de los docentes y del ambiente educativo, es que los valores más altos se observan en los primeros años y éstos van disminuyendo con el avance de la carrera. También resalta que los valores más bajos en las dimensiones de percepción de aprendizaje y autopercepción académica se observan en tercer año, y lo mismo sucede en sexto año en las dimensiones de percepción de los docentes y del ambiente. 
Tabla 1. Población de estudiantes y participantes en la encuesta de diagnóstico de ambiente educativo.

\begin{tabular}{|c|c|c|c|c|c|c|c|}
\hline \multirow{2}{*}{\multicolumn{2}{|c|}{ Variables }} & \multicolumn{6}{|c|}{ Participantes } \\
\hline & & Año I & Año II & Año III & Año IV & Año V & Año VI \\
\hline \multicolumn{2}{|l|}{ Participantes } & 96 & 72 & 76 & 107 & 70 & 66 \\
\hline \multicolumn{2}{|l|}{$\begin{array}{l}\text { Total matriculados* } \\
\text { Porcentaje de participación }\end{array}$} & $\begin{array}{c}118 \\
81.35 \%\end{array}$ & $\begin{array}{c}78 \\
92.3 \%\end{array}$ & $\begin{array}{c}113 \\
67.2 \%\end{array}$ & $\begin{array}{c}117 \\
91.45 \%\end{array}$ & $\begin{array}{c}92 \\
76.08 \%\end{array}$ & $\begin{array}{c}77 \\
85.7 \%\end{array}$ \\
\hline \multicolumn{2}{|l|}{ Sexo: mujeres (\%) } & 53,13 & 58,33 & 55,26 & 42,99 & 48,57 & 68,12 \\
\hline \multicolumn{8}{|l|}{ Edad (años) } \\
\hline & Mediana & 18 & 19 & 20 & 21 & 23 & 23 \\
\hline & Mínima - Máxima & $16-27$ & $17-33$ & $18-31$ & $19-30$ & $20-30$ & $21-28$ \\
\hline & Rango intercuartil & 4 & 8 & 6 & 8 & 6 & 5 \\
\hline
\end{tabular}

Dificultad percibida de la carrera

Comparado con otras carreras

$\begin{array}{rc}\text { Más fácil } & 0,41 \\ \text { Igual } & 2,45 \\ \text { Más difícil } & 89,80 \\ \text { No sabe } & 7,35\end{array}$

Comparado con medicina en otras universidades

$\begin{array}{rc}\text { Más fácil } & 0,41 \\ \text { Igual } & 5,51 \\ \text { Más difícil } & 81,43 \\ \text { No sabe } & 12,65\end{array}$

* Matriculados según registro de la Oficina de Admisiones y Registro.

En la Tabla 3 se observa las actividades extracurriculares, resaltando que antes del ingreso a estudiar medicina existe una gran mayoría de estudiantes que realizaban diversas actividades. Luego de ingresar a estudiar, es evidente una diminución de la realización de este tipo de actividades, siendo las que menos se alteran: Tocar un instrumento musical y asistir a misa o culto religioso. En la Tabla 4 se encuentran las asignaturas que se consideran más difíciles, que por mucho resultan ser Morfofisiología I (segundo semestre del primer año) y Farmacología I (primer semestre del tercer año); también es notorio que la mayoría corresponden al área de ciencias básicas. Estas mismas asignaturas fueron identificadas como las que más reprobación tienen (Tabla 5).

\section{Discusión}

El principal hallazgo que se encontró fue el cambio significativo con relación a la percepción de aprendizaje y autopercepción académica en tercer y sexto año, quizás debido a la inexperiencia en el manejo de pacientes e inicio del ejercicio médico dentro del contexto académico-asistencial, respectivamente, lo que implica tomar decisiones de acuerdo a los conocimientos propios y experticia. Llama la atención la disminución en la percepción de los docentes y del ambiente a lo largo de la carrera, probablemente se deba a que en la medida que el estudiante avanza en la carrera, es más crítico sobre la calidad de los docentes, en semestres superiores, es decir cuando mayor conocimiento médico poseen, sin embargo, hay que resaltar que en los primeros semestres, se podria dar la posibilidad que los estudiantes teman que la información pueda filtrarse y tener represalias como consecuencia, de esta forma prefieren no dar información al respecto.

Con respecto a la sensación del estudiante en la que percibe que estudiar medicina en la UIS es más difícil que en otras universidades, probablemente esté relacionado a que el proceso de selección de los aspirantes es más exigente y riguroso y sólo los altos puntajes de las pruebas Icfes Saber pro, pueden ingresar.

Se observó que en el aspecto de actividades extracurriculares antes y después del ingreso al plan de estudios de medicina en la UIS, ocurre un cambio significativo y prefieren no participar en ellas muy 
probablemente debido a que las actividades académicas ocupan la mayoría del tiempo, sacrificando el esparcimiento. Sin embargo el tocar algún instrumento musical o asistir a culto religioso, son las actividades que menos se afectan al estudiar medicina, probablemente debido a que la convicción religiosa y el don musical, cuando son verdaderos y forman parte de la identidad de la persona, se convierten en irrenunciables.

Tabla 2. Ambientes de aprendizaje en la Escuela de Medicina de la Universidad Industrial de Santander, 2018.

\begin{tabular}{|c|c|c|c|c|c|c|}
\hline \multirow[b]{2}{*}{ Año } & & \multicolumn{5}{|c|}{ Dimensiones del ambiente educativo } \\
\hline & & $\begin{array}{l}\text { Percepción del } \\
\text { Aprendizaje }\end{array}$ & $\begin{array}{l}\text { Percepción de } \\
\text { los docentes }\end{array}$ & $\begin{array}{c}\text { Autopercepción } \\
\text { Académica }\end{array}$ & $\begin{array}{l}\text { Percepción del } \\
\text { ambiente }\end{array}$ & $\begin{array}{c}\text { Autopercepción } \\
\text { social }\end{array}$ \\
\hline \multirow[t]{5}{*}{ I } & Media & 41,93 & & 28,66 & $43,35^{*}$ & 24,27 \\
\hline & Mediana & 43 & & 29 & 44 & 25 \\
\hline & Desviación estándar & 5,30 & ND & 4,40 & 6,25 & 4,59 \\
\hline & Mínimo & 25 & & 18 & 26 & 13 \\
\hline & Máximo & 52 & & 39 & 56 & 34 \\
\hline \multirow[t]{5}{*}{ II } & Media & $39,78^{*}$ & & 28,01 & $42,66^{*}$ & $23,24 *$ \\
\hline & Mediana & 40 & & 27 & 43 & 23 \\
\hline & Desviación estándar & 5,05 & ND & 5,25 & 6,40 & 3,63 \\
\hline & Mínimo & 29 & & 12 & 20 & 12 \\
\hline & Máximo & 51 & & 40 & 56 & 32 \\
\hline \multirow[t]{5}{*}{ III } & Media & $37,43^{*}$ & $38,11^{*}$ & 26,99 & $39,03 *$ & $22,16^{*}$ \\
\hline & Mediana & 37 & 38 & 27 & 40 & 22 \\
\hline & Desviación estándar & 5,71 & 5,67 & 3,80 & 6,33 & 4,49 \\
\hline & Mínimo & 21 & 24 & 20 & 18 & 10 \\
\hline & Máximo & 48 & 51 & 39 & 51 & 31 \\
\hline \multirow[t]{5}{*}{ IV } & Media & $39,22 *$ & $38,07^{*}$ & 28,82 & $40,13^{*}$ & $22,07 *$ \\
\hline & Mediana & 40 & 38 & 29 & 40 & 22 \\
\hline & Desviación estándar & 5,70 & 5,80 & 4,06 & 6,24 & 4,52 \\
\hline & Mínimo & 24 & 25 & 18 & 22 & 13 \\
\hline & Máximo & 53 & 53 & 38 & 58 & 35 \\
\hline \multirow[t]{5}{*}{$\mathrm{V}$} & Media & 39,23 & $35,16^{*}$ & 29,86 & $39,43^{*}$ & $23,67^{*}$ \\
\hline & Mediana & 39 & 35 & 30 & 41 & 24 \\
\hline & Desviación estándar & 6,60 & 5,79 & 4,61 & 7,50 & 3,89 \\
\hline & Mínimo & 20 & 22 & 16 & 20 & 12 \\
\hline & Máximo & 51 & 50 & 40 & 54 & 31 \\
\hline \multirow[t]{6}{*}{ VI } & Media & 38,07 & $34,31^{*}$ & 28,21 & $38,10^{*}$ & $22,34 *$ \\
\hline & Mediana & 39 & 35 & 30 & 39 & 23 \\
\hline & Desviación estándar & 5,25 & 4,59 & 4,73 & 6,39 & 3,74 \\
\hline & Mínimo & 20 & 26 & 15 & 25 & 10 \\
\hline & Máximo & 48 & 43 & 38 & 51 & 30 \\
\hline & Valor $\mathrm{p}:^{\mathrm{b}}$ & $<0,001$ & $<0,001^{\mathrm{c}}$ & 0,0013 & $<0,001^{\mathrm{c}}$ & 0,0007 \\
\hline
\end{tabular}

* con distribución normal según prueba de Shapiro-Wilk; ${ }^{\text {p }}$ prueba de Kruskal-Wallis; c prueba t de Student. ND: Datos no disponibles; los participantes no respondieron esos ítems. 
Tabla 3. Actividades extracurriculares de los estudiantes de pregrado de medicina de la Universidad Industrial de Santander, 2018.

\begin{tabular}{|c|c|c|c|c|}
\hline \multirow{2}{*}{ Actividades extracurriculares } & \multirow{2}{*}{$\begin{array}{l}\text { Antes de ingresar a } \\
\text { estudiar medicina }\end{array}$} & \multicolumn{3}{|c|}{ Frecuencia de realización durante los estudios de medicina $(\%)$} \\
\hline & & Disminuyó & Igual & Aumentó \\
\hline Leer libros & 84,60 & 65,91 & 12,60 & 21,49 \\
\hline Asistir a misa/culto religioso & 60,46 & 49,79 & 47,46 & 2,75 \\
\hline Jugar en el computador & 67,90 & 64,47 & 33,83 & 1,70 \\
\hline Salir a fiestas o conciertos & 61,03 & 59,66 & 28,79 & 11,55 \\
\hline Asistir a cine & 88,34 & 70,43 & 21,56 & 8,01 \\
\hline Practicar deporte & 78,69 & 72,14 & 19,75 & 8,11 \\
\hline Salir de paseo & 89,96 & 79,67 & 18,07 & 2,26 \\
\hline Salir a hacer compras & 77,94 & 65,00 & 31,25 & 3,75 \\
\hline Tocar instrumento musical & 36,51 & 45,71 & 53,00 & 1,29 \\
\hline Ver televisión & 82,41 & 79,71 & 17,81 & 2,48 \\
\hline
\end{tabular}

Tabla 4. Indicadores de relevancia (salience de Smith), obtenidos por listados libres, indicando las asignaturas más difíciles del pregrado de medicina en la UIS.*

\begin{tabular}{lccc}
\hline Asignatura & $\begin{array}{c}\text { Frecuencia } \\
(\%)\end{array}$ & $\begin{array}{c}\text { Posición } \\
\text { promedio }\end{array}$ & $\begin{array}{c}\text { Salience de } \\
\text { Smith }\end{array}$ \\
\hline Morfofisiología I & 79,7 & 1,02 & 0,792 \\
Farmacología I & 92,8 & 1,89 & 0,600 \\
Cirugía & 31,9 & 2,95 & 0,125 \\
Farmacologia II & 14,5 & 2,90 & 0,057 \\
Biociencias II & 4,3 & 2,00 & 0,032 \\
Medicina Interna & 5,8 & 2,75 & 0,028 \\
Patología & 2,9 & 2,50 & 0,016 \\
Biología Celular & 1,4 & 1,00 & 0,014 \\
Morfofisiología II & 1,4 & 2,00 & 0,010 \\
\hline
\end{tabular}

* Datos obtenidos exclusivamente de los estudiantes de internado. Los valores posibles están entre cero y uno, siendo mayor cuando más consenso hay.

Tabla 5. Indicadores de relevancia (salience de Smith), obtenidos por listados libres, indicando las asignaturas más reprobadas del pregrado de medicina en la UIS.*

\begin{tabular}{lccc}
\hline Asignatura & $\begin{array}{c}\text { Frecuencia } \\
(\%)\end{array}$ & $\begin{array}{c}\text { Posición } \\
\text { promedio }\end{array}$ & $\begin{array}{c}\text { Salience } \\
\text { de Smith }\end{array}$ \\
\hline Morfofisiología I & 65,0 & 1,08 & 0,630 \\
Farmacología I & 33,9 & 1,59 & 0,258 \\
Biología Celular & 8,9 & 1,13 & 0,084 \\
Patologia & 10,6 & 1,58 & 0,081 \\
Genética & 7,2 & 1,38 & 0,058 \\
Biociencias II & 5,0 & 2,00 & 0,031 \\
Morfofisiología II & 3,9 & 2,14 & 0,022 \\
Farmacologia II & 2,8 & 1,80 & 0,019 \\
Comunicación Científica & 0,6 & 4,00 & 0,001 \\
\hline
\end{tabular}

* Datos obtenidos exclusivamente de los estudiantes de internado. Los valores posibles están entre cero y uno, siendo mayor cuando más consenso hay.
Las asignaturas que se reprueban en mayor proporción corresponden a Morfofisiologia I y Farmacología general, cuestión que coincide con la percepción de las asginaturas más difíciles, lo cual puede estar relacionado con la exigencia y carga académica, por ello ameritaría continuar con las modificaciones del contenido, enfoque y estrategias educativas-evaluativas, de dichas asignaturas, para hacerlas más prácticas y de mayor utilidad para el futuro profesional. En el caso de cirugía general la percepción del estudiante, es de complejidad como asignatura, pero no se presenta un alto índice de reprobación, debido a la multievaluación que existe en la asignatura, lo cual le permite al estudiante oportunidad para aprobarla; aún así no se descartaría una modificación de su contenido buscando el mayor provecho y pertinencia para el médico general en formación.

En Colombia el DREEM ha sido usado en la Universidad de la Sabana para comparar la clase magistral con la estrategia de aula invertida, encontrando que esta última se presenta con un mejor ambiente educativo ${ }^{11}$.

El ambiente educativo de la Escuela de Medicina de la UIS es similar al encontrado en Universidades como Xavier University School of Medicine, Aruba; Shankar, et al. ${ }^{12}$ coinciden con nosotros en afirmar que la percepción de los docentes y ambiente, a medida que el estudiante avanza, disminuye sustancialmente, quizá por la imagen del maestro como autoritario y ridiculizador ${ }^{12}$; de la misma forma se halló en dicha universidad que los estudiantes especialmente en los primeros años de estudio, tienen dificultades para hacer frente a la exigencia académica en las materias del ciclo básico, cuestión que concuerda con el alto grado 
de reprobación de Morfofisiología y Farmacología, pertenecientes al ciclo básico, en la escuela de medicina de la UIS.

Kim, et al. ${ }^{13}$ refieren en su análisis, sobre ambiente educativo realizado en College of Korean Medicine, Kyung Hee University, que los estudiantes demuestran estrés en la vida universitaria e insatisfacción con el plan de estudios, hecho que se ve reflejado en el abandono de sus actividades extracurriculares ${ }^{13}$, situación similar encontrada en nuestro estudio.

Ríos, et al. ${ }^{16}$ muestran bajos puntajes en la autopercepción social, en los estudiantes de Latinoamérica (Paraguay, Venezuela y Colombia), en segundo, tercer y cuarto año, cuestión similar con lo encontrado en la escuela de Medicina de la Universidad Industrial de Santander, en dichos años, sin embargo, en cuarto año es cuando menos puntaje se encuentra. (Ver Tabla 2).

Es interesante ver que en los estudios revisados, incluido el nuestro, ninguno ha llegado a tener un ambiente educativo en general bueno.

La experiencia aquí descrita es valiosa para poder hacer seguimiento a los procesos de cambio curricular e innovación pedagógica futuros. El uso del DREEM actualmente se mantiene como actividad anual permanente de la evaluación del ambiente educativo, en varias universidades del mundo.

\section{Conclusiones}

Este estudio pretende ser herramienta útil para replantear la estructura y detectar probables debilidades del programa de medicina de pregrado en la UIS, con el objetivo generar recomendaciones a directivos, que permitan proyectar al estudiante de medicina como un ser integral y propender por el desarrollo armonico de sus dimensiones, en la que el aspecto académico sea considerado con igual importancia como el tiempo dedicado al descanso y a la practica de actividades extracurriculares, que le garanticen aprender sin afectar su calidad de vida.

El papel sustantivo de la labor docente es determinante para orientar el proceso académico, en el empalme educativo y clínico, el cual requiere un abordaje mediante una perspectiva general, en la que se visualice al estudiante como ser humano en proceso de aprendizaje, que requiere atención, para que elabore su propio conocimiento a partir de su experiencia ${ }^{14}$.

Confiamos en que nuestros resultados inspiren a directivos, a realizar mejoras en el currículo y sistemas de apoyo para responder a las necesidades dentro del entorno educativo, del estudiante de medicina de la Universidad Industrial de Santander.

\section{Agradecimientos}

A todos los estudiantes que participaron en el estudio. Al doctor Alvaro Javier Idrovo Velandia, Director de la Escuela de Medicina, por su apoyo irrestricto durante la realización de este trabajo y la asesoría en los análisis estadísticos.

\section{Referencias}

1. Bhutta ZA, Chen L, Cohen J, Crisp N, Evans T, Fineberg $\mathrm{H}$, et al. Education of health professionals for the 21st century: a global independent Commission. Lancet. 2010; 375(9721): 1137-1138. doi: 10.1016/S0140-6736(10)60450-3.

2. Prideaux D. The global-local tension in medical education: turning 'think global, act local' on its head? Med Educ. 2018 (in press). doi: 10.1111/ medu.13630.

3. Quintero GA. Medical education and the healthcare system - why does the curriculum need to be reformed? BMC Medicine. 2014; 12: 213. doi: https://doi.org/10.1186/s12916-014-0213-3.

4. Comisión para la Transformación de la Educación Médica en Colombia. Documento de recomendaciones para la transformación de la educación médica en Colombia. Bogotá DC: Ministerio de Salud y Protección Social / Ministerio de Educación; 2017.

5. Asociación Colombiana de Facultades de Medicina (Ascofame). Consenso de Montería.

6. Dent John-Harden Ronal. A Practical Guide for medical teachers. Fourth edition; 2013. Elsevier.

7. Aguilar-Barojas S, Jiménez-Sastré A, CastilloOrueta ML. Validación de la traducción al idioma español del Dundee Ready Education Environment Measure. Invest Educ Med; 2017 (in press). doi: http://dx.doi.org/10.1016/j.riem.2017.03.001.

8. Riquelme A, Oporto M, Oporto J, Méndez JI, Viviani $\mathrm{P}$, Salech F, et al. Measuring students' perceptions of the educational climate of the new curriculum at the Pontificia Universidad Católica de Chile: Performance of the Spanish translation of the Dundee 
Ready Education Environment Measure (DREEM). Educ Health (Abingdon). 2009; 22(1): 112.

9. Thompson EC, Juan Z. Comparative cultural salience: measures using free-list data. Field Methods. 2006; 18: 398-412. doi: http://dx.doi. org/10.1177/1525822X0101300303.

10. Smith JJ. Using ANTHROPAC 3.5 and a spreadsheet to compute a free-list salience index. Field Methods. 1993; 5(3): 1-3. doi: https://doi. org/10.1177/1525822X9300500301.

11. Domínguez LC, Vega NV, Espitia EL, Sanabria ÁE, Corso C, Serna AM, et al. Impacto de la estrategia de aula invertida en el ambiente de aprendizaje en cirugía: una comparación con la clase magistral. Biomedica. 2015; 35(4): 513-521. doi: 10.7705/ biomedica.v35i4.2640.

12. Shankar PR, Dubey AK, Balasubramanium R. Students'perception of the learning environment at Xavier University School of Medicine, Aruba. J Educ Eval Health Prof. 2013; 10(8): 1-5. doi: http:// dx.doi.org/10.3352/jeehp.2013.10.8.

13. Hyunho K, Hanyoung J, Pyeongjin J, Seungju K, Young-Bae P, Yeonseok K. Perception study of traditional korean medical students on the medical education using the Dundee Ready Educational Environment Measure. Evidence-Based Complement Alternat Med. 2016; 6042967: 1-5. doi: http://dx.doi.org/10.1155/2016/6042967.

14. Morales Gómez A, Medina Figueroa AM. Percepción del alumno de pregrado de Medicina, acerca del ambiente educativo. Rev Med Inst Seguro Soc. 2007; 45(2): 123-131.

15. Ortega Javier, Pérez C, Ortiz L, Fasce E, McColl $\mathrm{P}$, Torres G, et al. Estructura factorial de la escala DREEM en estudiantes de medicina Chilenos. Rev Med Chile. 2015; 143(5): 651-657. doi: http:// dx.doi.org/10.4067/S0034-98872015000500013.

16. Ríos González CM. Percepción del ambiente educativo en estudiantes de Medicina de Latinoamérica, 2015. CIMEL. 2016; 21(2): 38-41. 